\title{
Htlv1 Associated Myelopathy, Its Diagnosis, Management and Complications
}

\author{
Ghazaleh Shoja E Razavi* \\ Department of Clinical Development- Oncology and Respiratory, Global Allied Pharmaceutical, 160 Vista Oak Dr.Longwood, FL 32779, USA
}

\section{Editorial}

Human T-Cell Lymphotropic Virus Type 1 is the first discovered human retrovirus that has been isolated in 1979 from a patient with cutaneous T-Cell lymphoma. HTLV is a member of Retroviridae family in the genus Deltaretrovirus. Retroviruses are RNA viruses that produce DNA from RNA utilizing the enzyme reverse transcriptase. The DNA is subsequently incorporated into the host's genome. HTLV predominantly affects T lymphocytes [1,2].

Although HTLV-1 is found throughout the world, its global and loco-regional prevalence is not clear. Interestingly, this loco-regional prevalence is not similar even in the areas with high endemicity rate. Clusters of high endemicity has been reported in southwestern parts of Japan, parts of the Caribbean area, foci in South America, such as Colombia and French Guyana, some areas of intertropical Africa (such as South Gabon), specific parts of the middle East (north east of Iran, i.e. Mashhad and Neyshabur), and rare isolated clusters in Melanesia. Moreover, there are clusters of highly endemic areas, located often nearby areas where the virus is nearly absent. There are evidence that its distribution follows a geographic pattern rather that the ethnicity. In general, the prevalence is about $1-6 \%$ in endemic areas, however, this sero-prevalence is gradually increased with age, approaching to $25-40 \%$ in population elder than 50 in the highly endemic areas. Besides, many seropositive cases remain asymptomatic throughout their lives without any clinical manifestation and are not discovered through regular medical visits and laboratory evaluations [3-6].

HTLV1 is transmitted at least through three recognized ways. Its sexual transmission is mostly from male to female and correlates with increased risk of sero-positivity in females with increasing age. Blood products that are contaminated with HTLV1 infected lymphocytes are another potential source of infection. The third way is its vertical transmission from mother to infant that is mainly linked to breast feeding after the age of 6 months. Contaminated blood products and breast feeding source of transmission account for $15-60 \%$ seropositivity in transfusion recipients and $10-25 \%$ of breast fed infants of HTLV1 infected mothers, respectively [7-9].

Despite the variety in routs of exposure with variable risk of HTLV1 infection; fortunately, more than $90 \%$ of HTLV1 positive cases remain asymptomatic throughout their lives. Around 10 to 20 million HTLV1 infected cases are estimated worldwide; however, it is still unclear why a small percentage of them presented with the virus associated clinical manifestations. HTLV1 might cause 4 distinct clinical presentations; HTLV1 associated lymphoproliferative disorders (ATLL), HTLV1 associated Myelopathy/ Tropical Spastic Paraparesis (HAM/TSP), HTLV1 associated ocular disease which is mainly presented as uveitis, and cutaneous manifestations. All of these HTLV1 associated disorders present with different incidence and variable latency period among sero-positive population. ATLL; as an example, presents in around $1-4 \%$ of HTLV 1 carriers. The HTLV1 associated myelopathy is even less frequent than hematologic complications with the incidence of $1-2 \%$, respectively. The latency period for HTLV1 associated lymphoma has been reported to be as long as 20-30 years disorders. Similarly, HTLV1 associated myelopathy has a prolonged latency period of 20-30 years. However, this neurologic disorder with short latency period of 3 months has been reported in rare cases $[4,10]$. Co-incidence of HTLV1 associated myelopathy and lymphoproliferative disorders has not been reported.

From clinical point of view, the neurologic presentation of HTLV1 infection is a chronic inflammatory reaction of both gray and white matter in spinal cord with perivascular demyelination and axonal degeneration. It has a slowly progressive course with involvement of sensory, motor and autonomic nerves. Unlike the hematologic manifestation that shows a male predominance, the neurologic manifestation of HTLV1 is mostly reported in female patients and the average age of 40 at the time of presentation. The disease primarily presents with weakness in the lower extremities, sensory impairment with burning and tingling sensation, lumbar pain. In some cases however, urinary symptoms as well as sexual complaints might precede the weakness and sensory symptoms [11-14].

HAM/TSP is basically a chronic inflammatory disorder with a progressive nature. Indeed, the interaction between HTLV-I-infected CD4+ T cells and HTLV-I-specific CD8+ cytotoxic T cells (CTL), plays a critical role in the pathogenesis of HAM/TSP, through a longstanding bystander mechanism, that eventually lead to the destruction of surrounding nervous tissues. Altered interaction between CD4+ and $\mathrm{CD} 8+\mathrm{T}$ lymphocytes, as well as cytokine production seems to play a key role in its pathophysiology [15-17].

Clinical studies have shown that CD4+ T cells in the peripheral blood of HAM/TSP patients, particularly HTLV-I-infected CD4+ T cells, have an exaggerated transmigrating activity with the ability to accumulate in the tissues such as central nervous system. It has also been observed in several studies that the pro-viral load in the peripheral blood samples of HAM/TSP patients is significantly higher than asymptomatic HTLV1 infected cases. Moreover, examination of the cerebrospinal fluid (CSF) of HAM/TSP patients has been reported higher percentage of HTLVI-infected cells in CSF compared with peripheral blood mononuclear cells (PBMC) with a pro-viral load ratio of more than $10 \%$ in CSF vs. more than $1 \%$ in peripheral blood mononuclear cells, respectively. This ratio has been reported to be less than $10 \%$ (CSF) and less than $1 \%$ (PBMC) in asymptomatic HTLV1 carriers in comparative studies [1820].

Diagnosis of HAM/TSP is based on the clinical signs and symptoms of upper motor neuron lesions, such as spasticity, Babinski sign and

*Corresponding author: Ghazaleh Shoja E Razavi, Department of Clinical Development- Oncology and Respiratory, Global Allied Pharmaceutical, 160 Vista Oak Dr.Longwood, FL 32779, USA, Tel: 1- 416-520-8835; E-mail: ghazal966@gmail.com

Received September 10, 2015; Accepted September 12, 2015; Published September 18, 2015

Citation: Razavi GSE (2015) Htlv1 Associated Myelopathy, Its Diagnosis Management and Complications. Int $\mathrm{J}$ Neurorehabilitation 2: e111. doi:10.4172/2376-0281.1000e111

Copyright: () 2015 Razavi GSE. This is an open-access article distributed under the terms of the Creative Commons Attribution License, which permits unrestricted use, distribution, and reproduction in any medium, provided the original author and source are credited. 
hyperreflexia, neuropathic components and/or autonomic dysfunction, detection of HTLV1 in serum and CSF of the patients and exclusion of other differential diagnoses. Imaging technics such as brain and spinal cord MRI might be normal in spite of the presence of clinical symptoms; however, in advanced cases of HAM/TSP, Brain MRI might show periventricular and subcortical white matter alterations. The high signal intensity and the contrast enhancement are located mainly in the posterior columns, posterior horns, or lateral columns at the cervical or thoracic levels. However, the white matter lesions can be indistinguishable from those of other demyelinating diseases [21,22].

Multiple therapeutic approaches has been proposed and tried for HAM/TSP based on the inflammatory nature of the disorder and the prominent role of HTLV1 in its development. However, the disease is not curable and its progressive condition is generally associated with significant morbidity and compromised quality of life. Interferons, corticosteroids, and even chemotherapeutic agents targeting lymphocytes as the main component of the inflammatory reaction, such as mitoxantrone and cyclophosphamide and even plasmapheresis has been tried with some degrees of improvement. However, immunosuppressive treatments should be administered cautiously based on the altered immune response that is usually observed in HTLV1 infected patients. Anti-retroviral treatment with a combination of two nucleoside analogues such as zidovudine and lamivudine has also been suggested in selected patients with limited proviral loads and early clinical features, although clinical trials with anti-retroviral combination treatment was not impressive in advanced cases with severe autonomic and motor neuropathies. Mogamulizumab, a humanized monoclonal antibody targeting the chemokine receptor CCR4, that has recently been proposed and tried in phase I and II clinical trials in heavily pretreated patients with peripheral $\mathrm{T}$ cell lymphoma, mycosis fungoides (MF) and Sézary syndrome has also shown to be effective in decreasing the viral preload, as well as reducing inflammatory reaction associated with HAM/TSP. However, further clinical studies are needed to show its clinical efficacy and introduce the subgroup of the patients that might benefit from this treatment modality. Interestingly, valprioc acid has been proposed for the treatment of HAM/TSP based on the observations suggesting its anti-inflammatory and viral proliferation inhibitory role. However, in spite of its successful role in reducing the pro-viral load, valproic acid alone didn't show clinical benefit for HAM/TSP. Combination of valproic acid and anti-retroviral nucleoside analogue zidovudine also tried in animal models infected with simian T cell Lymphotrophic Virus 1 (STLV1) with resultant reduction in proviral load, but its clinical benefit in human infected with HTLV1 needs to be determined [23-28].

Apart from therapeutic approaches to control the disease, rehabilitation and supportive care is an important part of the management in HAM/TSP patients. Sensory, motor and autonomic involvement in these patients is associated with a wide range of signs and symptoms, such as neuropathic pain, dysesthesia, pin and needle sensation, motor deficits, bladder dysfunction, constipation and sexual complaints. Physiotherapy has been proposed as a palliative modality to reduce motor symptoms, pain and optimize the quality of life. Neurogenic bladder is mainly due to detrusor over-activity and/ or detrusor sphincter dyssynergia. It is the most common autonomic dysfunction observed in HAM/TSP patients with a frequency of $30 \%$ in different studies. It might precede other neurologic signs or symptoms in HAM/TSP patients. Different modalities such as anticholinergics and intermittent self-catheterization have been suggested for these patients. However, the risk of infection, bladder diverticula and bladder stones are significantly high among these patients. Low back pain is a prominent symptom in HAM/TSP patients that has been reported in up to $75 \%$ of patients. It is exacerbated with movements and interferes with patients' physical activity and has a significant negative impact in their quality of life. Analgesics, antidepressants, gabapentin and even corticosteroids have been proposed as a potential treatment for this pain with different efficacy [29-31].

HTLV1 is a retrovirus capable of causing a wide range of clinical manifestations, with at least two distinct categories of lymphoproliferative disorder and inflammatory myelopathy. The pathophysiology of these two clinical presentations, as well as the therapeutic approach and prognosis are quite different. Fortunately, among over 20 million sero-positive cases of HTLV1 worldwide, less than $5 \%$ of this infected population is at risk of these clinical diseases. Why these cases are prone to the clinical disease while a vast majority remains asymptomatic healthy infected throughout their lives is a puzzle, however, early acquisition of the virus during the childhood and high pro-viral load are among the possible etiologies. HAM/ TSP is a chronic inflammatory myelopathy with sensory, motor and autonomic components that more commonly affect women. Different therapeutic approaches have been suggested and tried in these patients with different responsiveness. However, the disease causes morbidity in many people in endemic areas of the world. Apart from therapeutic approach, supportive care and rehabilitation is an important clinical need in this group of patients. Novel approaches such as cannabinoids with their anti-spastic, analgesic, and immune modulatory effects might be beneficial for symptomatic management of these patients. Further clinical studies are mandated to address these unmet clinical needs.

\section{References}

1. Gallo RC (2005) The discovery of the first human retrovirus: HTLV-1 and HTLV2. Retrovirology 2: 17.

2. Poiesz BJ, Ruscetti FW, Gazdar AF, Bunn PA, Minna JD, et al. (1980) Detection and isolation of type $C$ retrovirus particles from fresh and cultured lymphocytes of a patient with cutaneous T-cell lymphoma. Proc Natl Acad Sci USA 77: 74157419 .

3. Blattner WA, Nomura A, Clark JW, Ho GY, Nakao Y, et al. (1986) Modes of transmission and evidence for viral latency from studies of human T-cel lymphotrophic virus type I in Japanese migrant populations in Hawaii. Proc Natl Acad Sci U S A 83: 4895-4898.

4. Proietti FA, Carneiro-Proietti AB, Catalan-Soares BC, Murphy EL (2005) Global epidemiology of HTLV-I infection and associated diseases. Oncogene 24: 6058-6068.

5. Rafatpanah H, Hedayati-Moghaddam MR, Fathimoghadam F, Bidkhori HR, Shamsian SK, et al. (2011) High prevalence of HTLV-I infection in Mashhad Northeast Iran: a population-based seroepidemiology survey. J Clin Virol 52 172-176.

6. Gessain A, Cassar O (2012) Epidemiological Aspects and World Distribution of HTLV-1 Infection. Frontiers in Microbiology 3:388.

7. Roucoux DF1, Wang B, Smith D, Nass CC, Smith J, et al. (2005) A prospective study of sexual transmission of human T lymphotropic virus (HTLV)-I and HTLVII. J Infect Dis 191: 1490-1497.

8. Hino S, Yamaguchi $\mathrm{K}$, Katamine $\mathrm{S}$, Sugiyama $\mathrm{H}$, Amagasaki T, Kinoshita $\mathrm{K}$, et al. (1985) Mother-to-child transmission of human T-cell leukemia virus type-I. Jpn. J. Cancer Res. 76: 474-480.

9. Inaba S, Sato H, Okochi K, Fukada K, Takakura F, et al. (1989) Prevention of transmission of human T-lymphotropic virus type 1 (HTLV-1) through transfusion, by donor screening with antibody to the virus. One-year experience. Transfusion 29: 7-11.

10. Yasunaga J, Matsuoka M (2007) Human T-cell leukemia virus type I induces adult T-cell leukemia: from clinical aspects to molecular mechanisms. Cancer Control 14: 133-140.

11. Cooper SA, van der Loeff MS, Taylor GP (2009) The neurology of HTLV-1 infection. Pract Neurol 9: 16-26. 
Citation: Razavi GSE (2015) Htlv1 Associated Myelopathy, Its Diagnosis, Management and Complications. Int J Neurorehabilitation 2: e111. doi:10.4172/2376-0281.1000e111

12. De Castro-Costa CM, Araújo AQ, Barreto MM, Takayanagui OM, Sohler MP, da Silva EL, et al. (2006) Proposal for diagnostic criteria of tropical spastic paraparesis/HTLV-I-associated myelopathy (TSP/HAM). AIDS Res. Hum. Retroviruses 22: 931-935.

13. Oliveira P, Castro NM, Carvalho EM (2007) Urinary and sexual manifestations of patients infected by HTLV-I. Clinics (Sao Paulo) 62: 191-196.

14. Orland JR, Engstrom J, Fridey J, Sacher RA, Smith JW, et al. (2003) Prevalence and clinical features of HTLV neurologic disease in the HTLV Outcomes Study. Neurology 61: 1588-1594.

15. Osame M (2002) Pathological mechanisms of human T-cell lymphotropic virus type I-associated myelopathy (HAM/TSP). J Neurovirol 8: 359-364.

16. ljichi S, Izumo S, Eiraku N, Machigashira K, Kubota R, Nagai M, et al. (1993) An autoaggressive process against bystander tissues in HTLV-I-infected individuals: a possible pathomechanism of HAM/TSP. Med Hypotheses 41: 542-547.

17. Nakamura T (2009) HTLV-I-associated myelopathy/tropical spastic paraparesis (HAM/TSP): the role of HTLV-I-infected Th1 cells in the pathogenesis, and therapeutic strategy. Folia Neuropathol 47: 182-194.

18. Lezin A, Olindo S, Olie're S, Varrin-Doyer M, Marlin R, Cabre P, et al. (2005) Human T Lymphotropic Virus Type I (HTLV-I) Proviral Load in Cerebrospinal Fluid: A New Criterion for the Diagnosis of HTLV-I-Associated Myelopathy/ Tropical Spastic Paraparesis? J Infect Dis 191: 1830-1834

19. Nagai M, Yamano Y, Brennan MB, Mora CA, Jacobson S (2001) Increased HTLV-I proviral load and preferential expansion of HTLV-I Tax-specific CD8+ T cells in cerebrospinal fluid from patients with HAM/TSP. Ann Neurol 50: 807812.

20. Nakamura T, Furuya T, Nishiura Y, Ichinose K, Shirabe S, et al. (2000) Importance of immune deviation toward Th1 in the early immunopathogenesis of human T-lymphotropic virus type I-associated myelopathy. Med Hypotheses 54: 777-782.

21. Lezin A, Olindo S, Oliere S, Varrin-Doyer M, Marlin R, et al. (2005) Human T lymphotropic virus type I (HTLV-I) proviral load in cerebrospinal fluid: a new criterion for the diagnosis of HTLV-I-associated myelopathy/tropical spastic paraparesis? J Infect Dis 191: 1830-1834.
22. Morgan DJ, Caskey MF, Abbehusen C, Oliveira-Filho J, Araujo C, et al. (2007) Brain magnetic resonance imaging white matter lesions are frequent in HTLV-I carriers and do not discriminate from HAM/TSP. AIDS Res Hum Retroviruses 23: $1499-1504$.

23. Izumo S, Goto I, Itoyama Y, Okajima T, Watanabe S, et al. (1996) Interferonalpha is effective in HTLV-I-associated myelopathy: a multicenter, randomized, double-blind, controlled trial. Neurology 46: 1016-1021.

24. Taylor GP, Goon P, Furukawa Y, Green H, Barfield A, et al. (2006) Zidovudine plus lamivudine in Human T-Lymphotropic Virus type-I-associated myelopathy: a randomised trial. Retrovirology 3: 63.

25. Duvic M, Pinter-Brown LC2, Foss FM3, Sokol L4, Jorgensen JL1, et al. (2015) Phase 1/2 study of mogamulizumab, a defucosylated anti-CCR4 antibody, in previously treated patients with cutaneous T-cell lymphoma. Blood 125: 18831889.

26. Yamauchi J, Coler-Reilly A, Sato T, Araya N, Yagishita N, Ando H, et al. (2015) Anti-CCR4 antibody mogamulizumab targets human T-lymphotropic virus type I-infected CD8+ as well as CD4+ T cells to treat associated myelopathy. J Infect Dis. 211:238-248.

27. Olindo S, Belrose G, Gillet N, Rodriguez S, Boxus M, et al. (2011) Safety of long-term treatment of HAM/TSP patients with valproic acid. Blood 118: 63066309.

28. Willems L (2009) The 14th International Conference on Human Retrovirology: HTLV and related retroviruses (July 1-4, 2009; Salvador, Brazil). Retrovirology 6: 77.

29. Sá KN, Macêdo MC, Andrade RP, Mendes SD, Martins JV, et al. (2015) Physiotherapy for human T-lymphotropic virus 1-associated myelopathy: review of the literature and future perspectives. Journal of Multidisciplinary Healthcare 8:117-125.

30. Tannus M, Tanajura D, Sundberg MA, Oliveira P, Castro N, et al. (2011) Detrusor Arreflexia as an End Stage of Neurogenic Bladder in HAM/TSP? Case Rep Med 2011: 289389.

31. Tavares IR, Franzoi AC, Araújo AQ (2010) Low-back pain in HTLV-I-associated myelopathy/tropical spastic paraparesis: nociceptive or neuropathic? Spinal Cord 48: 134-137. 